\title{
Convergence Almost Everywhere of Non-convolutional Integral Operators in Lebesgue Spaces
}

\author{
Yakhshiboev M. U. \\ Faculty of Mathematics, National University of Uzbekistan, Tashkent, 100174, Tashkent, Uzbekistan
}

\begin{abstract}
Received September 2, 2020; Revised November 8, 2020; Accepted November 19, 2020
Cite This Paper in the following Citation Styles

(a): [1] Yakhshiboev M. U. , "Convergence Almost Everywhere of Non-convolutional Integral Operators in Lebesgue Spaces," Mathematics and Statistics, Vol. 8, No. 6, pp. 705 - 710, 2020. DOI: 10.13189/ms.2020.080611.

(b): Yakhshiboev M. U. , (2020). Convergence Almost Everywhere of Non-convolutional Integral Operators in Lebesgue Spaces. Mathematics and Statistics, 8(6), 705 - 710. DOI: 10.13189/ms.2020.080611.
\end{abstract}

Copyright $@ 2020$ by authors, all rights reserved. Authors agree that this article remains permanently open access under the terms of the Creative Commons Attribution License 4.0 International License

\begin{abstract}
The case of one-dimensional and multidimensional non-convolutional integral operators in Lebesgue spaces is considered in this paper. The convergence in the norm and almost everywhere of non-convolution integral operators in Lebesgue spaces was insufficiently studied. The kernels $K_{\varepsilon}(x, y)$ of non-convolutional integral operators do not need to have a monotone majorant, so the well-known results on the convergence almost everywhere of convolutional averages are not applicable here. The kernels $K_{\varepsilon}(x, y)$ of nonconvolutional integral operators take into account different behaviors at $|y| \rightarrow 0$ and $|y| \rightarrow \infty$ depending on $\varepsilon \rightarrow 0$ (which is important in applications) and cover the situation in the particular case of convolutional and non-convolutional integral operators. We are interested in the behavior of function $K_{\varepsilon} \varphi$ as $\varepsilon \rightarrow 0$. Theorems on convergence almost everywhere in the case of one-dimensional and multidimensional nonconvolution integral operators in Lebesgue spaces are proved. The theorems proved are more general ones (including for convolutional integral operators) and cover a wide class of kernels.
\end{abstract}

Keywords Non-convolutional Integral Operator, Convergence Almost Everywhere, Chen-Marchaud Fractional Derivatives, Non-convolutional Averaging

\section{Introduction}

A modification of Liouville fractional integro-differentiation on a straight line $R$ is considered in [6], "attached" to a certain fixed point $c \in R,|c|<\infty$, and convenient in a way that it can be applied to functions set on the entire straight line that can have any increment at infinity:

$$
\left(I_{c}^{\alpha} \varphi\right)(x):=\left\{\begin{array}{c}
\frac{1}{\Gamma(\alpha)} \int_{c}^{x} \frac{\varphi(t)}{(x-t)^{1-\alpha}} d t, x>c, \\
\frac{1}{\Gamma(\alpha)} \int_{x}^{c} \frac{\varphi(t)}{(t-x)^{1-\alpha}} d t, x<c,
\end{array}\right.
$$

where $x \in R, \alpha>0$. Apparently, fractional integration in this form first was mentioned in (Chen Y.W. [1]). The corresponding fractional differentiation in the Riemann-Liouville form was also considered there

$$
\left(D_{c}^{\alpha} f\right)(x):=\frac{1}{\Gamma(1-\alpha)}\left\{\begin{aligned}
\frac{d}{d x} \int_{c}^{x} \frac{f(t)}{(x-t)^{\alpha}} d t, & x>c, \\
-\frac{d}{d x} \int_{x}^{c} \frac{f(t)}{(t-x)^{\alpha}} d t, & x<c,
\end{aligned}\right.
$$

where $x \in R, 0<\alpha<1$. Fractional integration

$D_{c}^{\alpha} f=\frac{1}{\aleph(\alpha, l)} \int_{0}^{\infty} \frac{\left(\Delta_{t}^{l} f_{c+}\right)(x)-\left(\Delta_{-t}^{l} f_{c-}\right)(x)}{t^{1+\alpha}} d t, l>\alpha>0$

in the form of Marchaud was considered by A.V. Skorikov in ([11]), where it was used to describe space $L_{p}^{\alpha}(\Omega)$ (restricted on $(a, b)$ Bessel potentials on $R$ ). A number of properties of Chen fractional integro-differentiation can be found in ([7], Section 18.5, [9]).

In [3], [6], three different ways of reduction of the ChenMarchaud constructions for fractional differentiation of $D_{c}^{\alpha}$, are considered, and these different options for reduction are used to describe and invert the fractional integrals (1) of functions from $L_{p}^{l o c}(R)$. Three different ways of "truncating" the fractional derivative $D_{c}^{\alpha}$, give different integral representations for the truncated Chen-Marchaud fractional derivatives. 
The resulting integral representations are operators that approximate the unit operator. The first integral representation falls under the influence of the well-known theorems on convolutional approximations of unity, and the operators of the second and third integral representations of a non-convolutional structure require a special study. Non-convolutional integral operators in space $L_{p}(R)$ were poorly studied. In this regard, one class of non-convolutional averaging is considered in [8]

$$
\left(A_{\varepsilon} f\right) \quad(x)=
$$

$=\int_{0}^{\frac{1}{\varepsilon_{1}}} \ldots \int_{0}^{\frac{1}{\varepsilon_{n}}} k_{1}\left(y_{1}\right) \ldots k_{1}\left(y_{n}\right) \varphi(x-\varepsilon \circ y \circ(x-c)) d y_{1} \ldots d y_{n}$,

where $c \in R^{n}, x-\varepsilon \quad \circ \quad y \quad \circ \quad(x-c)=$ $\left(x_{1}-\varepsilon_{1} y_{1}\left(x_{1}-c_{1}\right), \ldots, x_{n}-\varepsilon_{n} y_{n}\left(x_{n}-c_{n}\right)\right)$,

$0<\varepsilon_{i}<1, \int_{0}^{\infty} k_{i}\left(y_{i}\right) d y_{i}=1, i=\overline{1, n}$ and the convergence to the function $\varphi(x)$ in norm $L_{\bar{p}}\left(R^{n}\right)$ (or $L_{\bar{p}}^{\text {loc }}\left(R^{n}\right)$ ) is investigated.

Non-convolutional integral representations in Lebesgue space were obtained in [13]. Operator (2) is not convolutional and kernels $k_{i}(t), i=\overline{1, n}$, do not need to have a monotone majorant, so the well-known results on convergence of convolutional averages almost everywhere ([12], p. 77-78) are not applicable here. We will set simple conditions ensuring the convergence of construction (2) to $\varphi(x)$ almost everywhere, having obtained them as a corollary of the statement on a nonconvolutional operator convergence almost everywhere.

Paper [2] argued strong convergence and $\Delta$-convergence theorems for the class of generalized non-expansive multivalued maps in a CAT(0) space. Furthermore, Paper [10] presented different methods for bandwidth selection in bivariate kernel density estimation based on the principle of gradient method and compare the result with the biased cross-validation method. Also, the asymptotic mean integrated squared error is used as the measure of performance of the new methods.

In this paper, multi-dimensional non-convolutional integral operators are studied

$$
\left(K_{\varepsilon} \varphi\right)(x)=\int_{R^{n}} K_{\varepsilon}(x, y) \varphi(x-y) d y, x \in R^{n}
$$

in space $L_{p}\left(R^{n}\right)$. In the case when the kernel $K_{\varepsilon}(x, y)$, in (3) do not depend on $x$, some theorems on convergence almost everywhere of similar averages with an arbitrary dependence on $\varepsilon$ are known, (see, for example, U. Neri [5], p.16 or T Kurokawa [4]). However, even for this convolutional case, the assumptions about the kernel are restrictive in these works.

The kernel $K_{\varepsilon}(x, y)$ in (3) takes into account different behaviors as $|y| \rightarrow 0$ and $|y| \rightarrow \infty$ depending on $\varepsilon \rightarrow 0$ (which is important in applications) and covers the situation of operator (2). We are interested in the behavior of function $K_{\varepsilon} \varphi$ as $\varepsilon \rightarrow 0$.

In the proof of this theorem, we follow, to a large extent, the proof of Theorem 2.8 (T.Kurokawa [4]), where convolutional averaging was considered; our condition 3) in Theorem 2 being proved is much more general (including the convolutional operators) and covers a wide class of kernels.

In this paper, we prove the convergence almost everywhere of the non-convolutional integral operator (3) in space $L_{p}\left(R^{n}\right)$.

\section{Materials and methods}

The research material is the object in the case of onedimensional and multidimensional non-convolutional integral operators. The methods of differential and integral calculus, as well as the methods of functional analysis are used in the study.

\section{Auxiliary statements}

Let $f(x)$ be locally integrable and $W(x, \varepsilon)=$ $\{y:|y-x| \leq \varepsilon\}$ is the ball of radius $\varepsilon$ centered at point $x$. According to Lebesgue's theorem

$$
\frac{1}{\operatorname{mes} W(x, \varepsilon)} \int_{W(x, \varepsilon)} f(y) d y \rightarrow f(x)
$$

almost everywhere on $R^{n}$.

Definition 1. Point $x\left(x \in R^{n}\right)$ is called the Lebesgue function $f(x)$, if

$$
\frac{1}{\operatorname{mes} W(x, \varepsilon)} \int_{W(x, \varepsilon)}|f(y)-f(x)| d y \rightarrow 0
$$

as $\varepsilon \rightarrow 0$.

It is clear that any point of continuity of function $f(x)$ is a Lebesgue point for $f(x)$.

Remark 1. Note that Definition 1 implies that if $x\left(x \in R^{n}\right)$ is a Lebesgue point, then

$$
\lim _{\varepsilon \rightarrow 0} \frac{1}{\varepsilon^{n}} \int_{|y| \leq \varepsilon}|f(x-y)-f(x)| d y=0 .
$$

\section{Main results}

\subsection{The case of one-dimensional non- convolutional integral operators}

Consider the non-convolution integral operator

$$
\left(K_{\varepsilon} \varphi\right)(x)=\int_{-\infty}^{\infty} k_{\varepsilon}(x, y) \varphi(x-y) d y, x \in R .
$$

Theorem 1. Let $\varphi(x) \in L_{p}\left(R^{1}\right), 1 \leq p \leq \infty$ and let for any sufficiently small $\mu>0$, the following conditions hold: 
1) $\int_{|y|<\mu} k_{\varepsilon}(x, y) d y \rightarrow 1$ as $\varepsilon \rightarrow 0$ for almost all $x$;

2) $\int_{|y|>\mu}\left|k_{\varepsilon}(x, y)\right|^{p^{\prime}} d y \rightarrow 0$, if $p>1$ and

$\sup \left|k_{\varepsilon}(x, y)\right| \rightarrow 0$, if $p=1$, as $\varepsilon \rightarrow 0$ for almost all $x$;

$|y|>\mu$

3) There are numbers $\alpha>0, \beta>0$ and almost everywhere finite functions are $a(x)>0, c(x)>0$, such that at $|y|<\mu$

$$
\left|k_{\varepsilon}(x, y)\right| \leq\left\{\begin{array}{c}
c(x)|y|^{\alpha-1} \cdot \varepsilon^{-\alpha}, \text { at }|y| \leq a(x) \cdot \varepsilon \\
c(x)|y|^{-\beta-1} \varepsilon^{\beta}, \text { at }|y|>a(x) \cdot \varepsilon
\end{array}\right.
$$

Then

$$
\lim _{\varepsilon \rightarrow 0}\left(K_{\varepsilon} \varphi\right)(x)=\varphi(x)
$$

holds for almost all $x$ (functions $\varphi(x)$ at Lebesgue points, if functions $a(x), c(x)$ are finite at these points).

If $k_{\varepsilon}(x, y) \equiv 0$ at $|y| \geq N=N(x)$ for any sufficiently small $\varepsilon$, then (5) is true for any function $\varphi(x) \in$ $L_{p}^{l o c}\left(R^{1}\right), 1 \leq p \leq \infty$.

Proof. Fix a point $x$, considering it the Lebesgue point of function $\varphi(x)$. Let $h$ be an arbitrary small number $h>0$. Let us show that

$$
\left|\left(K_{\varepsilon} \varphi\right)(x)-\varphi(x)\right|<c h
$$

by choosing $\varepsilon=\varepsilon(h)$ where $c$ does not depend on $h$ and $\varepsilon$ (but may depend on $x$ ).

For simplicity of further notation, consider the case when $k_{\varepsilon}(x, y)=0$ for $y<0$, based on the fact that the case when $k_{\varepsilon}(x, y) \equiv 0$ for $y>0$ is considered completely similar due to the "evenness" of the theorem conditions with respect to $y$.

We have

$$
\left|\left(K_{\varepsilon} \varphi\right)(x)-\varphi(x)\right| \leq I_{1}+I_{2}+I_{3},
$$

where

$$
\begin{gathered}
I_{1}=\int_{0}^{\mu}\left|k_{\varepsilon}(x, y)\right||\varphi(x-y)-\varphi(x)| d y, \\
\left.I_{2}=\mid \int_{0}^{\mu} k_{\varepsilon}(x, y) d y-1\right)|| \varphi(x) \mid, \\
I_{3}=\int_{\mu}^{\infty}\left|k_{\varepsilon}(x, y)\right||\varphi(x-y)| d y,
\end{gathered}
$$

$\mu$ while $\mu>0$ is arbitrary. Since $x$ is the Lebesgue point, we can indicate such $\mu>0$ that

$$
\frac{1}{y} \int_{0}^{y}|\varphi(x-\tau)-\varphi(x)| d \tau<h, 0<y \leq \mu .
$$

Assume that $\mu$ is chosen so small that (7) and condition 3) of Theorem 1 hold. Now let's fix $\mu=\mu(h)=\mu(h, x)$. Using condition 1) of Theorem 1, we obtain

$$
I_{2} \leq c_{2} h
$$

by choosing $\varepsilon=\varepsilon(h, \mu)$, for $c_{2}=|\varphi(x)|$. The application of Holder's inequality in $I_{3}$ gives

$$
\begin{aligned}
& I_{3}: \leq\left(\int_{\mu}^{\infty}\left|k_{\varepsilon}(x, y)\right|^{p^{\prime}} d y\right)^{\frac{1}{p^{\prime}}} \times \\
& \times\left(\int_{|y|>\mu}|\varphi(x-y)|^{p} d y\right)^{\frac{1}{p}} \leq C_{3} h
\end{aligned}
$$

with condition 2) of Theorem 1 (also by choosing $\varepsilon=\varepsilon(h, \mu)$ ), $C_{3}=\|\varphi(x)\|_{L_{p}(R)}$.

It remains to determine $I_{1}$. We have:

$$
\begin{aligned}
I_{1} & :=\int_{0}^{a(x) \varepsilon}\left|k_{\varepsilon}(x, y)\right||\varphi(x-y)-\varphi(x)| d y+ \\
& +\int_{a(x) \varepsilon}^{\mu}\left|k_{\varepsilon}(x, y)\right||\varphi(x-y)-\varphi(x)| d y
\end{aligned}
$$

assuming that $\varepsilon$ is chosen so small that $0<a(x) \varepsilon<\mu$. Using assumption 3) of Theorem 1, we obtain:

$$
\begin{gathered}
I_{1} \leq \frac{c(x)}{\varepsilon^{\alpha}} \int_{0}^{a(x) \cdot \varepsilon}|y|^{\alpha-1}|\varphi(x-y)-\varphi(x)| d y+ \\
+c(x) \cdot \varepsilon^{\beta} \int_{a(x) \cdot \varepsilon}^{\infty}|y|^{-\beta-1}|\varphi(x-y)-\varphi(x)| d y=I_{1}^{\prime}+I_{1}^{\prime \prime} .
\end{gathered}
$$

Denote by $G(y)=G_{x}(y):=\int_{0}^{y}|\varphi(x-\tau)-\varphi(x)| d \tau$, so that

$$
G(y) \leq h y, 0<y<\mu
$$

according to (7). Integrating by parts we have:

$$
\begin{aligned}
I_{1}^{\prime}= & \frac{c(x)}{\varepsilon^{\alpha}}\left\{y^{\alpha-1} G(y) \mid \begin{array}{c}
y=a(x) \varepsilon \\
y=0
\end{array}\right. \\
& \left.-(\alpha-1) \int_{0}^{a(x) \cdot \varepsilon} y^{\alpha-2} G(y) d y\right\} .
\end{aligned}
$$

With (10) we obtain $\left.y^{\alpha-1} G(y)\right|_{y=0}=0$. Using (10) with (11) considering that $a(x) \cdot \varepsilon<\mu$ we have

$$
I_{1}^{\prime} \leq c_{1}^{\prime} h
$$

where $c_{1}^{\prime}=\left[1+\frac{|\alpha-1|}{\alpha}\right] c(x) a(x)^{\alpha}$. Similar integration by parts in $I_{1}^{\prime \prime}$ gives:

$$
I_{1}^{\prime \prime}=c(x) \cdot \varepsilon^{\beta}\left\{y^{-\beta-1} G(y) \mid \begin{array}{c}
y=\mu \\
y=a(x) \varepsilon
\end{array}+\right.
$$




$$
\left.+(\beta+1) h \int_{a(x) \cdot \varepsilon}^{\mu} y^{-\beta-2} G(y) d y\right\} .
$$

Using (10) again, we obtain:

$$
I_{1}^{\prime \prime} \leq \frac{1+2 \beta}{\beta} c(x) h\left[a(x)^{-\beta}+\left(\frac{\varepsilon}{\mu}\right)^{\beta}\right] .
$$

Since $\varepsilon<\frac{\mu}{a(x)}$, hence

$$
I_{1}^{\prime \prime} \leq c_{1}^{\prime \prime} h
$$

where $c_{1}^{\prime \prime}=\frac{2}{\beta}(1+2 \beta) \frac{c(x)}{a(x)^{\beta}}$. Collecting inequalities (8), (9), (12), and (13), we obtain the required estimate (6).

It remains to add that the intersection of the set of Lebesgue points with the set of points where $a(x)$ and $c(x)$ are finite, has full measure.

Finally, if the kernel $k_{\varepsilon}(x, y)$ is finite in terms of $y$, then estimate (9) is the only one where the information that $\varphi(x) \in$ $L_{p}(R)$-function $\varphi(x)$ is integrated over a finite segment was used, so it is suffice that $\varphi(x) \in L_{p}^{\text {loc }}(R)$.

\subsection{The case of multidimensional convolutional integral operators}

Theorem 2. Let $\varphi(x) \in L_{p}\left(R^{n}\right), 1 \leq p \leq \infty$ and let the following conditions be met for any sufficiently small $\mu>0$ :

1) $\int_{|y|<\mu} K_{\varepsilon}(x, y) d y \rightarrow 1$ as $\varepsilon \rightarrow 0$ for almost all $x$;

2) $\int_{|y|>\mu}\left|K_{\varepsilon}(x, y)\right|^{p^{\prime}} d y \rightarrow 0$, if $p>1$ and

$\sup \left|K_{\varepsilon}(x, y)\right| \rightarrow 0$, if $p=1$, as $\varepsilon \rightarrow 0$ for almost all $x$;

$|y|>\mu$

3) There are numbers $\alpha>0, \beta>0$ and finite function almost everywhere $a(x)>0, c(x)>0$, such that $|y|<\mu$

$$
\left|K_{\varepsilon}(x, y)\right| \leq\left\{\begin{array}{c}
c(x)|y|^{\alpha-1} \cdot \varepsilon^{-\alpha},|y| \leq a(x) \cdot \varepsilon, \\
c(x)|y|^{-\beta-1} \varepsilon^{\beta},|y|>a(x) \cdot \varepsilon .
\end{array}\right.
$$

Then

$$
\lim _{\varepsilon \rightarrow 0}\left(K_{\varepsilon} \varphi\right)(x)=\varphi(x)
$$

is true for almost all $x$.

If $K(x, y) \equiv 0$ for $|y| \geq N=N(x)$ for any sufficiently small $\varepsilon$, then (14) is true for all $\varphi(x) \in L_{p}^{\text {loc }}\left(R^{n}\right)$.

Proof. Let $x\left(x \in R^{n}\right)$ be the Lebesgue point of function $f(x)$. We have

$$
\left|\left(K_{\varepsilon} \varphi\right)(x)-\varphi(x)\right| \leq J_{1}+J_{2}+J_{3},
$$

where

$$
\begin{gathered}
\left.J_{1}:=\mid \int_{|y|<\mu} K_{\varepsilon}(x, y) d y-1\right)|| \varphi(x) \mid, \\
J_{2}:=\int_{|y|<\mu}\left|K_{\varepsilon}(x, y)\right||\varphi(x-y)-\varphi(x)| d y,
\end{gathered}
$$

$$
J_{3}:=\int_{|y|>\mu}\left|K_{\varepsilon}(x, y)\right||\varphi(x-y)| d y .
$$

By condition 1) of the Theorem 2 we get that $J_{1} \leq C_{1} h$ by choosing $J_{1} \leq C_{1} h$, here $C_{1}=|\varphi(x)|$.

Application of Holders inequality in $J_{3}$ gives:

$$
\begin{gathered}
J_{3}: \leq\left(\int_{y \mid>\mu}\left|K_{\varepsilon}(x, y)\right|^{p^{\prime}} d y\right)^{\frac{1}{p^{\prime}}}\left(\int_{|y|>\mu}|\varphi(x-y)|^{p} d y\right)^{\frac{1}{p}} \leq \\
\leq C_{3} h
\end{gathered}
$$

by condition 2) of Theorem 2 (and by choosing $\varepsilon=\varepsilon(h, \mu)$ ), $C_{3}=\|\varphi(x)\|_{L_{p}\left(R^{n}\right)}$.

It remains to estimate $J_{2}$. Using assumption 3 ) of the Theorem 2, we get

$$
J_{2} \leq I_{1}+I_{2}+I_{3}
$$

where

$$
I_{1}=\frac{c(x)}{\varepsilon^{\alpha}} \int_{|y|<a(x) \cdot \varepsilon}|y|^{\alpha-1}|\varphi(x-y)-\varphi(x)| d y,
$$

$$
\begin{gathered}
I_{2}=c(x) \cdot \varepsilon^{\beta} \int_{a(x) \cdot \varepsilon<|y|<\mu}|y|^{-\beta-1}|\varphi(x-y)-\varphi(x)| d y, \\
I_{3}=c(x) \cdot \varepsilon^{\beta} \int_{|y|>\mu}|y|^{-\beta-1}|\varphi(x-y)-\varphi(x)| d y .
\end{gathered}
$$

Introduce auxiliary function

$$
G(\rho)=G_{x}(\rho)=\int_{|y| \leq \rho}|\varphi(x-y)-\varphi(x)| d y .
$$

Since $x$ is the Lebesgue point, then according to (4), we have

$$
\lim _{\rho \rightarrow 0} \rho^{-n} G(\rho)=0 .
$$

So, setting arbitrary number $h>0$, we can determine $\mu>0$, so that $G(\rho) \leq h \rho^{n}$, for all $\rho \leq \mu$. Now estimate $I_{1}, I_{2}$, passing to spherical coordinates $|y|=\rho, \frac{y}{|y|}=y^{\prime}, d y=$ $\rho^{n-1} d r d y^{\prime}, y^{\prime}$ is the point on a unit sphere $\Sigma, d y^{\prime}$ is the element of the surface area of a unit sphere. We have

$$
\begin{aligned}
& I_{1} \leq \frac{c(x)}{\varepsilon^{\alpha}} \int_{\Sigma} d y^{\prime} \int_{0}^{a(x) \cdot \varepsilon} \rho^{\alpha+n-2}|\varphi(x-y)-\varphi(x)| d \rho= \\
& =\frac{c(x)}{\varepsilon^{\alpha}} \int_{0}^{a(x) \cdot \varepsilon} \rho^{\alpha-1} d \rho \int_{\Sigma} \rho^{n-1}|\varphi(x-y)-\varphi(x)| d y^{\prime}= \\
& =\frac{c(x)}{\varepsilon^{\alpha}} \int_{0}^{a(x) \cdot \varepsilon} \rho^{\alpha-1} d\left(\int_{0}^{\rho} \frac{d \rho}{r^{-n+1}} \int_{\Sigma}|\varphi(x-y)-\varphi(x)| d y^{\prime}\right)= \\
& =\frac{c(x)}{\varepsilon^{\alpha}} \int_{0}^{a(x) \cdot \varepsilon} \rho^{\alpha-1} d\left(\int_{y \mid \leq \rho}|\varphi(x-y)-\varphi(x)| d y\right)=
\end{aligned}
$$




$$
\begin{gathered}
=\frac{c(x)}{\varepsilon^{\alpha}} \int_{0}^{a(x) \cdot \varepsilon} \rho^{\alpha-1} d G(\rho), \\
I_{2} \leq c(x) \cdot \varepsilon^{\beta} \int_{\Sigma} d y^{\prime} \int_{a(x) \cdot \varepsilon}^{\mu} \rho^{-\beta+n-2}|\varphi(x-y)-\varphi(x)| d \rho= \\
=c(x) \cdot \varepsilon^{\beta} \int_{a(x) \cdot \varepsilon}^{\mu} \frac{d \rho}{\rho^{\beta+1}} \int_{\Sigma} \rho^{n-1}|\varphi(x-y)-\varphi(x)| d y^{\prime}= \\
\int_{a(x) \cdot \varepsilon}^{\mu} \frac{c(x) \varepsilon^{\beta}}{\rho^{\beta+1}} d\left(\int_{0}^{\rho} \frac{d \rho}{r^{-n+1}} \int_{\Sigma}^{\mu}|\varphi(x-y)-\varphi(x)| d y^{\prime}\right)= \\
\left.=\int_{a(x) \cdot \varepsilon}^{\mu} \frac{c(x) \cdot \varepsilon^{\beta}}{\rho^{\beta+1}} d \int_{y y \mid \leq \rho}^{\mu}|\varphi(x-y)-\varphi(x)| d y\right)= \\
=c(x) \cdot \varepsilon^{\beta} \int_{a(x) \cdot \varepsilon}^{\mu} \rho^{-\beta-1} d G(\rho) .
\end{gathered}
$$

Thus, the following equalities are true

$$
\begin{aligned}
& I_{1}=c(x) \cdot \varepsilon^{-\alpha} \int_{0}^{a(x) \cdot \varepsilon} \rho^{\alpha-1} d G(\rho), \\
& I_{2}=c(x) \cdot \varepsilon^{\beta} \int_{a(x) \cdot \varepsilon}^{\mu} \rho^{-\beta-1} d G(\rho),
\end{aligned}
$$

where $G(\rho)$ is taken from (15). Integrating (16) and (17) by parts we determine

$$
\begin{gathered}
I_{1}=\frac{c(x)}{\varepsilon^{\alpha}}\left\{\left.\rho^{\alpha-1} G(\rho)\right|^{\rho=a(x) \cdot \varepsilon}-\right. \\
\rho=0 \\
\left.-(\alpha-1) \int_{0}^{a(x) \cdot \varepsilon} \rho^{\alpha-2} G(\rho) d \rho\right\}, \\
I_{2}=c(x) \cdot \varepsilon^{\beta}\left\{\rho^{-\beta-1} \cdot G(\rho) \mid \rho=\mu(x) \cdot \varepsilon+\right. \\
\rho=a(j) \\
\left.+(\beta+1) \int_{a(x) \cdot \varepsilon}^{\mu} \rho^{-\beta-2} G(\rho) d \rho\right\} .
\end{gathered}
$$

By virtue of $G(\rho) \leq h \cdot \rho^{n}$ we obtain $\left.\rho^{\alpha-1} G(\rho)\right|_{\rho=0}=$ 0 . Using $G(\rho) \leq h \rho^{n}$ in (18), (19) considering that $a(x) \cdot \varepsilon<$ $\mu$, we have

$$
\begin{gathered}
I_{1} \leq \frac{c(x)}{\varepsilon^{\alpha}}\left\{\rho^{\alpha+n-1} h \mid \rho=a(x) \cdot \varepsilon-\right. \\
\left.-|\alpha-1| \cdot h \int_{0}^{a(x) \cdot \varepsilon} \rho^{\alpha+n-2} d \rho\right\} \leq
\end{gathered}
$$

$$
\begin{gathered}
\leq c(x) \cdot \varepsilon^{n-1}(a(x))^{\alpha+n-1}\left[1+\frac{|\alpha-1|}{\alpha+n-1}\right] h \leq C_{2}^{\prime} h, \\
I_{2}=c(x) \cdot \varepsilon^{\beta}\left\{\rho^{-\beta+n-1} \cdot h \mid \begin{array}{c}
\rho=\mu \\
\rho=a(x) \cdot \varepsilon
\end{array}+\right. \\
\left.+(\beta+1) h \int_{a(x) \cdot \varepsilon}^{+} \rho^{-\beta+n-2} d \rho\right\} \leq \\
\leq c(x) \cdot h\left\{\varepsilon^{\beta} \mu^{-\beta+n-1}-\varepsilon^{n-1}(a(x))^{-\beta+n-1}\right\} \times \\
\times\left[1+\frac{\beta+1}{\beta-n+1}\right] \leq C_{2}^{\prime \prime} h .
\end{gathered}
$$

Assume that $p=1$ (for $p>1$, the Hölder's inequality should be used in the estimation of integral $\left.\int_{|y|>\mu}|y|^{-\beta-1}|\varphi(x-y)| d y\right)$

$$
\begin{gathered}
I_{3}=c(x) \cdot \varepsilon^{\beta} \int_{|y|>\mu}|y|^{-\beta-1}|\varphi(x-y)-\varphi(x)| d y \leq \\
\leq c(x) \cdot \varepsilon^{\beta}\left\{\int_{|y|>\mu}|y|^{-\beta-1}|\varphi(x-y)| d y+\right. \\
\left.\left.\quad+|\varphi(x)| \int_{|y|>\mu}|y|^{-\beta-1} d y\right\}\right\} \leq \\
\leq c(x) \varepsilon^{\beta}\left\{\frac{1}{\mu^{\beta+1}}\|\varphi\|_{L_{1}}+\frac{m e s \Sigma}{\beta+1-n} \frac{1}{\mu^{\beta+1-n}}\right\} \leq C_{2}^{\prime \prime \prime} h .
\end{gathered}
$$

Note that in (20) $\mu$ is fixed. Since $\varphi(x)$ is locally integrable, $\varphi(x)$ is finite almost everywhere. Therefore, for any Lebesgue point, except maybe a set of measure zero, $|\varphi(x)|$ is a finite value. But then the right-hand side in (20) tends to zero as $\varepsilon \rightarrow 0$, i.e. $I_{3} \rightarrow 0$ as $\varepsilon \rightarrow 0$.

It remains to add that the intersection of the set of Lebesgue points with the set of points, where $a(x)$ and $c(x)$ are finite values, has full measure.

Finally, if the kernel $K_{\varepsilon}(x, y)$ is finite with respect to $y$, then in (3), at fixed $x$, only the local integrability of the function is used.

\section{Conclusions}

The importance of studying generalizations of nonconvolutional integral operators is due to their application in the theory of fractional integro-differentiation. The results obtained in this paper are sufficient conditions for the kernel $\varepsilon \rightarrow 0$ in non-convolutional integral operators (3), that consider different behavior at $|y| \rightarrow 0$ and $|y| \rightarrow \infty$ depending on $\varepsilon \rightarrow 0$, and cover the situation in the particular case of convolutional and non-convolutional integral operators.

\section{Acknowledgements}

We are very grateful to experts for their appropriate and constructive suggestions to improve this template. 


\section{REFERENCES}

[1] Chen Y.W. "Entire solutions of a class of differential equations of mixed type". Comm. Pure and Appl. Math., Vol.14, No.3. pp. 229-255, 1961.

[2] Fiaz Hussain, Saima Zainab "Strong and $\triangle-$ Convergence Results for Generalized Multi-valued Non-expansive Maps in CAT (0) Spaces". Mathematics and Statistics, Vol. 5, No.2, pp. 68-73, 2017. https://doi.org/10.13189/ms.2017.050203

[3] Humberto R. and Yakhshiboev M.U. "The Chen-Marchaud fractional integro-differentiation in the variable exponent Lebesgue spaces". Fract.calc. and Appl.Anal. Vol. 3, pp. 501$518,2011$.

[4] Kurokawa T. "On the Riesz and Bessel kernels as approximation of the identity". Sei. Repts Kagoshima univ., Vol. 30, pp. 31-45, 1981.

[5] Neri U. "Singular integrals". -Leet. Notes in Math. Vol.200, pp.1-272, 1971.

[6] Samko S.G. and Yakhshiboev M.U. "On one modification of Riemann-Liouville fractional integro-differentiation, applicable to functions on $R^{1}$ with any behavior at infinity". Universities Reports, Mathematics, No. 4, pp. 96-99, 1992.

[7] Samko S.G., Kilbas A.A. and Marichev O.I. "Fractional Integrals and Derivatives. Theory and Applications". London-New-
York: Gordon \& Breach. Sci. Publ., (Russian edition - "Fractional Integrals and Derivatives and some of their Applications", Minsk: Nauka i Tekhnika, 1987.), 1993, pp. 1-1012.

[8] Samko S.G., Yakhshiboev M.U. On a class of identity approximation operators of a non-convolution type. J. Fract. Calc. and Appl. Anal. Vol. 4, pp. 523-530, 2001.

[9] Samko S.G. and Yakhshiboev M.U. "A Chen-type Modification of Hadamard Fractional Integro-Dfferentiation", Operator Theory: Advances and Applications, Vol. 242, pp. 325-339, 2014.

[10] Siloko, I. U., Ishiekwene, C. C., Oyegue, F. O. New Gradient Methods for Bandwidth Selection in Bivariate Kernel Density Estimation. Mathematics and Statistics, Vol. 6, No. 1, pp. 1-8, 2018. https://doi.org/10.13189/ms.2018.060101

[11] Skorikov A.V. "To the description of spaces $L_{p}^{\alpha}(\Omega)$ ". Mat. Notes. Vol. 17, No. 5, pp. 691-701, 1975.

[12] Stein I. "Singular integrals and differential properties of functions". -M., Mir. 1973, p.1-342.

[13] Yakhshiboev M.U. "A Chen-type Modification of Ball Fractional Integro-Differentiation". Uzbek Mathematical Journal, No. 2, pp. 135-153, 2019. doi:10.29229/uzmj.2019-2-6 